\title{
The Effect of an Expanded Underwriting Portfolio on Premium and Capital
}

\author{
by Dongsae Cho*
}

\begin{abstract}
This paper demonstrates that growth of an underwriting portfolio produces a diversification effect, which justifies an increase in dividends to owners and a higher premium level for policyholders. Thus a transfer of wealth from policyholders to owners occurs. The results imply that the advantages of a mutual over a stock insurer are (i) protection of policyholders' wealth, (ii) benefit for policyholders from diversification, and (iii) an easier access to better quality insurance. Despite these advantages some policyholders still prefer a stock insurer because a mutual insurance company charges substantial amount of onetime membership fee upfront.
\end{abstract}

\section{Introduction}

It is well accepted among academicians, practitioners, and regulators that the amount of insurer capital required to maintain a given solvency probability level does not increase proportionately with the size of an underwriting portfolio. Instead, a proportional increase in capital is believed to improve its solvency probability. This benefit of expansion is anticipated not only when two or more portfolios are merged (see $[1,5])$, but also when insurance companies exchange reinsurance on a reciprocal basis (see $[1,2,3,4,12,15]$ ).

The foregoing belief, however, is subject to some major criticisms. First, from the perspective of the CAPM, mergers and reinsurance arrangements are redundant because owners of an insurer are in a position to diversify their investment portfolios. But this argument is ignored in this article, since in reality most owners do not possess sufficient financial

* Dongsae Cho is Assistant Professor of Insurance at the University of Minnesota in the Twin Cities. His article, "Applicability of Net Present Cost in Life Insurance Price Disclosure" published in The Journal of Insurance Issues and Practices in 1987, was nominated for the journal's Outstanding Article Award. The author appreciates the valuable suggestions of the two anonymous reviewers on earlier drafts of the paper. This project was supported with a research grant from the McKnight Foundation. 
resources to do so. Second, it will be of interest to examine this benefit when an underwriting portfolio expands in size not necessarily through mergers and reinsurance. Third, the impact of the size of a portfolio on premium needs to be explored as well, because solvency probability depends not only on capital but also on premium. Fourth, the benefit of growth occurs only when solvency probability of component groups exceeds a certain point under the assumption of near normally or normally distributed aggregate losses (see Venezian [16]).

The purpose of the article is, considering the above criticisms, to provide a complete analysis of the issue (i.e., relationship between the size of an underwriting portfolio and premium and capital) in more general and broader terms. First, the study examines the effect of adding one policyholder to an existing underwriting portfolio, which is more general than mergers or reinsurance. Second, the impact of expansion is measured both in terms of premium and capital. Third, general aggregate loss function is used rather than limiting the analysis to normally or near normally distributed aggregate losses $[1,13,16]$.

\section{Insurance premium structure}

In the context of the CAPM, the price of insurance depends upon how covered losses fluctuate compared to general market movement, because the owners of an insurer can diversify. In this article, however, an insurance premium is derived from the distribution of claims against an insurance company because most owners do not diversify. By assuming away administrative expenses and profit margin for an insurer, the price of insurance for a group of policyholders is simply the expected value of aggregate claims during a period. Because policyholders do not recover catastrophic losses in excess of the sum of premium and capital, expected aggregate losses depend upon the size of capital, and consequently upon the solvency probability level of the insurer.

Given this fact one can formulate an insurance premium at $1-\epsilon$ solvency probability level as follows.

$$
\Pi^{\epsilon}=\int_{0}^{\Pi^{\epsilon}+U^{\epsilon}} L h(L) d L+\left(\Pi^{\epsilon}+U^{\epsilon}\right) \int_{\Pi^{\epsilon}+U^{\epsilon}}^{\infty} h(L) d L,
$$

where $\Pi^{\epsilon}$ is insurance premium for all policyholders (hereafter, it is referred to as premium) to cover the policy period at the $1-\epsilon$ solvency probability level, $U \epsilon$ is insurer capital retained as a financial buffer at the $1-\epsilon$ probability level, $L$ is aggregate claims against the insurer (hereafter, loss), and $h(L)$ is probability density function of aggregate claims (hereafter, loss distribution). Equation (1) shows that premium is derived from a truncated loss distribution due to the limited liability of the insurance company.

In the case of a stock insurer, $U$ is supplied by stockholders in the beginning with part of earnings being added to it over time. For a mutual insurer, $U$ may be supplied in a number of ways: (i) founding policyholders may pay in the required capital, (ii) money may be borrowed, or (iii) a stock insurer may be converted into a mutual insurer after properly compensating stockholders for their ownership interest. After its inauguration or conversion, part of insurer earnings will be added to this account over time. 
Note that

$$
H\left(\Pi^{\epsilon}+U^{\epsilon}\right)=1-\epsilon,
$$

where $H$ is cumulative density function of $h .^{1}$ Using equation (2), one can compute $\Pi$ and $U$ at the $1-\epsilon$ solvency probability level from the cumulative probability distribution of loss as follows. $\Pi^{\epsilon}$ can be solved for from (1) without knowing $\Pi^{\epsilon}$ and $U^{\epsilon}$ because the level of $L$ at the 1- $\epsilon$ probability level can easily be identified from $H .^{2}$ Once $\Pi \epsilon$ is computed, calculation of $U^{\epsilon}$ is trivial because it is simply the difference between $\Pi^{\epsilon}$ and $L$ at the $1-\epsilon$ probability level. For notational convenience, superscript $\epsilon$ is omitted hereafter. Since premium depends upon solvency probability, it is necessary to assume a world of perfect knowledge at no cost. Information such as loss distribution and financial status of the insurer is available to all market participants free of charge. ${ }^{3}$ Also owners and policyholders are assumed to have homogeneous expectations with respect to future loss.

After some algebra, equation (1) becomes

$$
\begin{aligned}
\Pi & =L H(L) \mid \begin{array}{c}
\Pi+U \\
0
\end{array}-\int_{0}^{\Pi+U} H(L) d L+(\Pi+U)[1-H(\Pi+U)] \\
& =(\Pi+U) H(\Pi+U)-\int_{0}^{\Pi+U} H(L) d L+(\Pi+U)[1-H(\Pi+U)] \\
& =\Pi+U-\int_{0}^{\Pi+U} H(L) d L
\end{aligned}
$$

or equivalently

$$
U=\int_{0}^{\Pi+U} H(L) d L .
$$

Equation (4) shows how the fair amount of capital is calculated from $H$. Again it can be done without requiring any knowledge of $\Pi$ and $U$ because $H$ is known.

In this hypothetical insurance world, the following theorems may be derived.

Theorem 1: If policyholders pay more $\Pi$ for a given coverage, owners should also increase $U$ to achieve equity between the two interest groups.

\footnotetext{
${ }^{1}$ In reality solvency probability is affected not only by $\Pi$ and $U$ as implied by equation (2), but also by "nearly all the economical activities and decision making processes of an insurer". See Pentikainen [14].

2 Although the true $H$ is unknown in the real world, a number of researchers handled this problem by assuming that it is known (see Witt $[17,18]$ ), or by approximating $H$ using alternative methods (see $[1,7,9])$.

3 If one relaxes this assumption, positive transaction cost will increase the premium to $\Pi+a$ (where $a$ is transaction cost), while $U$ is unaffected.
} 
Proof: Differentiation of (4), after some algebra, produces

$$
\frac{d U}{d \Pi}=\frac{H(\Pi+U)}{1-H(I+U)}>0 . \quad \text { Q.E.D. }
$$

The above inequality implies that one dollar increase in $\Pi$ should be matched by $U$ amounting to $\$ \frac{H(\Pi+U)}{1-H(\Pi+U)}$. Because both $\Pi$ and $U$ have increased, the probability of solvency will $1-H(\Pi+U)$
also increase by $h(\Pi+U)\left(1+\frac{d U}{d \Pi}\right)$. The result may be interpreted as follows. An increased $U$ improves solvency probability of the insurance company or the quality of insurance. Naturally equity requires that policyholders pay more for the better quality insurance, thus creating a positive relationship between $I$ and $U$.

Theorem 2: As policyholders pay more $\Pi$, marginal increase in $U$ per unit of $\Pi(d U / d \Pi)$ should also increase.

Proof: By differentiating (5), we have

$$
\begin{aligned}
\frac{d^{2} U}{d \Pi^{2}}= & \frac{[1-H(\Pi+U)] h(\Pi+U)\left(1+\frac{d U}{d \Pi}\right)+H(\Pi+U) h(\Pi+U)\left(1+\frac{d U}{d \Pi}\right)}{[1-H(\Pi+U)]^{2}} \\
= & \frac{h(\Pi+U)\left(1+\frac{d U}{d \Pi}\right)}{[1-H(\Pi+U)]^{2}}>0 . \quad \text { Q.E.D. }
\end{aligned}
$$

From (5) and (6a), obviously $U$ monotonically increases in $\Pi$ at an increasing pace. The result implies that the relative contribution of owners compared to policyholders increases as the quality of insurance improves. Therefore owners have little incentives to maintain solvency probability beyond the required minimum. This issue is further discussed in section 5 .

\section{Diversification effect from the growth of an underwriting portfolio}

Consider an underwriting portfolio of $n$ policyholders with the following characteristics: (i) each policyholder has identical loss distribution with its mean and variance of $E(x)$ and $\operatorname{Var}(x)$, respectively, and (ii) loss to a policyholder is independent among the $n$ policyholders. The mean and variance of loss for the insurer then become

$$
\begin{aligned}
& E(L)_{n}=n E(x), \text { and } \\
& \operatorname{Var}(L)_{n}=n \operatorname{Var}(x),
\end{aligned}
$$

where $E(L)$ and $\operatorname{Var}(L)$ are the mean and variance of loss to the insurer, respectively, and subscripts $n$ or $n+1$, which will soon appear, denote the number of policyholders in the underwriting portfolio. If the insurance company adds to the portfolio another policyholder with the same loss characteristics, the mean and variance of loss would change to 


$$
\begin{aligned}
& E(L)_{n+1}=(n+1) E(x), \text { and } \\
& \operatorname{Var}(L)_{n+1}=(n+1) \operatorname{Var}(x) .
\end{aligned}
$$

For easier comparison of similar but different distributions, coefficient of variation (which measures the degree of dispersion relative to its mean) is often used. Coefficient of variation $(\alpha)$ for the above two distributions is given by

$$
\begin{aligned}
& \alpha_{n}=\sqrt{\frac{n \operatorname{Var}(x)}{n E(x)}}, \text { and } \\
& \alpha_{n+1}=\sqrt{\frac{\sqrt{(n+1) \operatorname{Var}(x)}}{(n+1) E(x)}}
\end{aligned}
$$

After some algebra, clearly

$$
\alpha_{n}>\alpha_{n+1}
$$

implying that, as an underwriting portfolio grows, the relative dispersion of loss decreases.

As the number of policyholders increases from $n$ to $n+1$, the shape of loss distribution will also change. Because the two portfolios hold different number of policyholders, it is necessary to make an adjustment to one of the two loss distributions for easier comparison. This adjustment can be accomplished by scaling down the new loss distribution for $n+1$ policyholders to $n$ policyholders. The scaled down distribution is constructed by reducing all levels of $L$ in the new distribution for $n+1$ policyholders to $n L /(n+1)$ while keeping corresponding probabilities unchanged.

Let the loss distribution of the original portfolio ( $n$ policyholders) and the new loss distribution ( $n+1$ policyholders) scaled down to $n$ be $g(L)$ and $f(L)$, respectively. Expected loss of $f$ is given by

$$
E(L)_{n+1}^{n}=\frac{n(n+1) E(x)}{n+1}=n E(x) .
$$

Superscript $n$ together with subscript $n+1$ indicates that the distribution is scaled down from $n+1$ to $n$ policyholders. These super and subscripts to $\Pi, U, M$, and $N$, which will appear later, will denote the same meaning. From (7) and (14), $f$ and $g$ have the same mean; from (13) $g$ has a greater coefficient of variation than $f$, therefore the standard deviation of loss for $g$ is greater than that of $f$.

The purpose of Figure 1 is to demonstrate that expansion of an underwriting portfolio produces a diversification effect by showing that $g$ is stochastically dominant in second order over $f$. 


\section{Figure 1}

Second Order Stochastic Dominance

of a Smaller Underwriting Portfolio

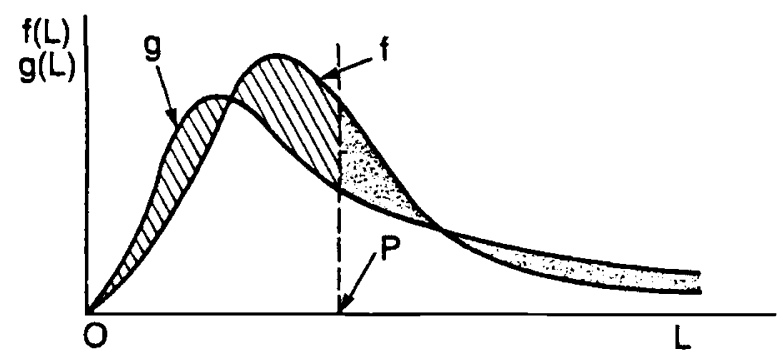

(a)

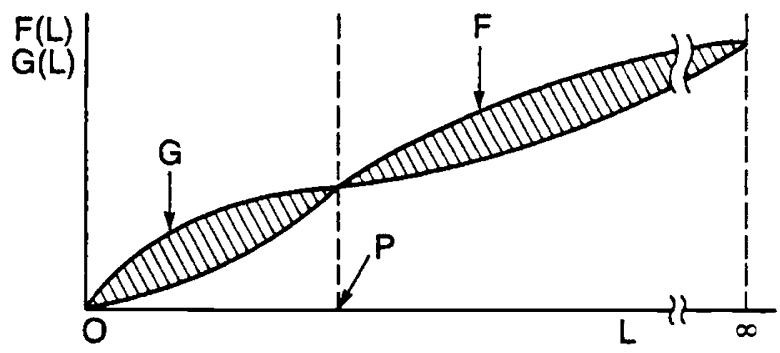

(b)

$\int_{0}^{L}[G(L)-F(L)] d L$

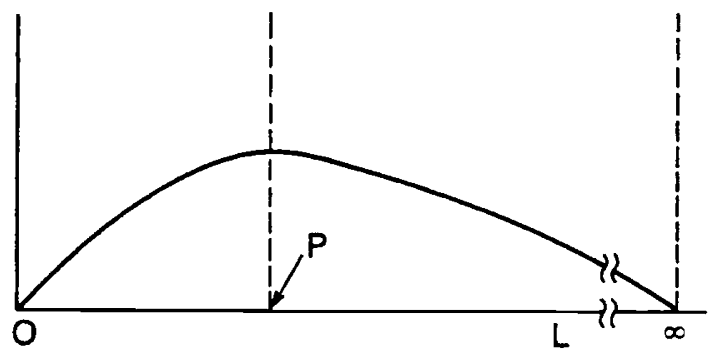

(c) 
It is impossible to give the definite shapes of $f$ and $g$, since only the mean and standard deviation are known. For this reason, $f$ and $g$ are arbitrarily patterned in Figure 1(a) using the available information (i.e., expected value and standard deviation of loss) under the assumption that they cross no more than twice. Although both $f$ and $g$ are drawn skewed to the right, it is unnecessary for the analysis that follows. Figure 1(b) presents the cumulative probability distributions of $f$ and $g$ or $F$ and $G$, respectively. Let us pick point $P$, which, if a vertical line is drawn from it, makes the two shaded areas in Figure 1(a) the same, and the two dotted areas in Figure 1(a) equivalent. At point $P$ in Figure 1(b), $F$ and $G$ intersect each other.

Expected loss for $f$ and $g$ may be rewritten as

$$
\begin{aligned}
& E(L)_{n}=\int_{0}^{\infty} L g(L) d L=\left.L G(L)\right|_{0} ^{\infty}-\int_{0}^{\infty} G(L) d L, \text { and } \\
& E(L)_{n+1}^{n}=\int_{0}^{\infty} L f(L) d L=\left.L F(L)\right|_{0} ^{\infty}-\int_{0}^{\infty} F(L) d L .
\end{aligned}
$$

Since $E(L)_{n}=E(L)_{n+1}^{n}$ from (7) and (14), the following relation holds.

$$
\int_{0}^{\infty} F(L) d L=\int_{0}^{\infty} G(L) d L,
$$

indicating that the shaded areas in Figure 1(b) below and beyond the intersection are the same.

Hence, for all levels of $L$,

$$
\int_{0}^{L}[G(L)-F(L)] d L \geq 0 .
$$

The above inequality is graphically presented in Figure 1(c). From the figure, it is clear that (i) the term is nonnegative for all values of $L$; (ii) it has a maximum at point $P$; and (iii) when $L=0$ or $\infty$, it is zero. Inequality (18), therefore, suggests that $g$ is stochastically dominant over $f$ in second order, implying a diversification effect from the growth of an underwriting portfolio.

\section{The impact of diversification on premium and capital}

Figure 2 superimposes on Figure 1(b) solvency probabilities of the insurer before (horizontal line $A A^{\prime}$ ) and after (horizontal line $A A^{\prime}, B B^{\prime}$, or $C C^{\prime}$, as the case may be) the growth. Horizontal line $A A^{\prime}$ ' may cross the upper shaded area, the intersection, or the lower shaded area. A rational insurer would not expand its underwriting portfolio for the case of Figure 2(c), because solvency probability decreases from $A A^{\prime}$ to $C C^{\prime}$ after the expansion at the corresponding level of fund. For this reason, this article focuses only on the cases of Figure 2(a) and 2(b). An additional assumption is also made for expositional convenience that the insurer is not allowed to dispose of the extra wealth (i.e., $Y-X$ in Figure 2(a)) created by diversification. Instead it must keep the extra wealth after the expansion to improve its solvency probability from $A A^{\prime}$ to $B B^{\prime}$. 
Figure 2

Impact of Diversification on Solvency Probability

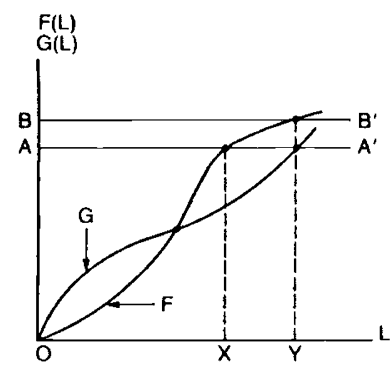

(a)

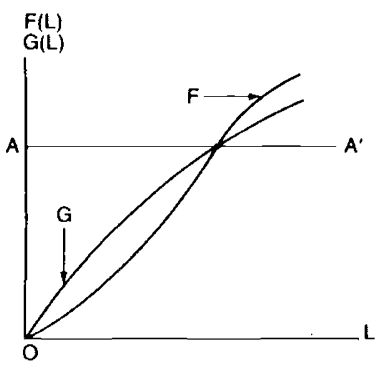

(b)

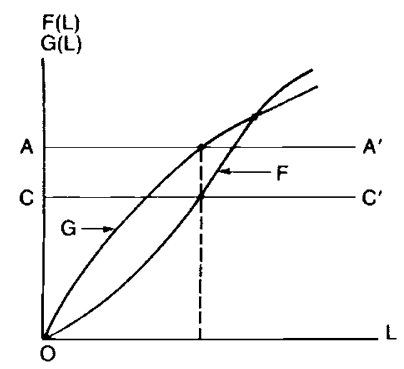

(c)

Theorem 3: Expanding an underwriting portfolio produces a diversification effect, and may improve solvency probability. But after the expansion dividends should be paid out to owners, and policyholders have to pay higher $\Pi$ to achieve fairness between the two groups.

Proof: The legal requirement that the insurer hold the same level of fund before and after the expansion yields the following relationship.

$$
\Pi_{n}+U_{n}=\Pi_{n+1}^{n}+U_{n+1}^{n}
$$

All elements of the above equation come directly from $f$ and $g$.

If $A A^{\prime}$ crosses the intersection (the case of Figure 2(b)), solvency probability of the insurer will remain the same before and after the expansion. In this case, the following relations are obtained from (4).

$$
\begin{gathered}
U_{n}=\int_{0}^{\Pi_{n}+U_{n}} G(L) d L, \text { and } \\
U_{n+1}^{n}=\int_{0}^{\Pi_{n+1}^{n}+U_{n+1}^{n}} F(L) d L .
\end{gathered}
$$

From Figure 2(b), clearly

$$
U_{n}>U_{n+1}^{n} .
$$

In view of (19) and (22),

$$
\Pi_{n+1}^{n}>\Pi_{n},
$$

implying that after the expansion owners of the insurer should receive dividends amounting to $U_{n}-U_{n+1}^{n}$, and that policyholders have to pay higher premium. 
If $A A^{\prime}$ ' crosses the upper shaded area (the case of Figure 2(a)), solvency probability will improve from $A A^{\prime}$ to $B B^{\prime}$ after the expansion because the insurer holds the extra wealth. In this case, relation (22) will hold up because $g$ is stochastically dominant over $f$ in second order, therefore relation (23) will also hold up. Hence, dividends should be paid to owners, and the insurer must charge higher $\Pi$ to policyholders after the expansion. Q.E.D.

Since the result is surprising and somewhat counterintuitive, the validity of theorem 3 is cross examined as follows. Let the first and second terms of the RHS of (1) be $M$ and $N$, respectively. If the insurer has $\$ P$ (the case of Figure 2(b)),

$$
\begin{aligned}
& M_{n+1}^{n}>M_{n}, \text { and } \\
& N_{n+1}^{n}=N_{n} .
\end{aligned}
$$

Therefore relation (23), and as a result (22), will hold up. If the insurer has more than $\$ P$ (the case of Figure 2(a)),

$$
\begin{gathered}
\Pi_{n}=E(L)_{n}-\int_{\Pi_{n}+U_{n}}^{\infty}\left[L-\left(\Pi_{n}+U_{n}\right)\right] g(L) d L, \text { and } \\
\Pi_{n+1}^{n}=E(L)_{n+1}^{n}-\int_{\Pi_{n+1}^{n}+U_{n+1}^{n}}^{\infty}\left[L-\left(\Pi_{n+1}^{n}+U_{n+1}^{n}\right)\right] f(L) d L .
\end{gathered}
$$

In view of $E(L)_{n}=E(L)_{n+1}^{\mathrm{n}}$ and (19), relations (23) and (22) will also hold up.

Theorem 3 indicates that the benefit of diversification from the growth of an underwriting portfolio goes to the owners of the insurer in the form of dividends. Policyholders should pay more for better quality insurance from diversification. Consequently the wealth of policyholders will transfer to owners, not in the sense that premium is unfair to policyholders, but in the sense that diversification costs more to policyholders.

\section{Implications}

One may infer from theorem 3 as to why the two major forms of insurance companies (stocks and mutuals) coexist in the private insurance market. A policyholder buying insurance from a mutual insurer pays $\left(\Pi_{n}+U_{n}\right) / n$ or $\left(\Pi_{n+1}^{n}+U_{n+1}^{n}\right) / n$, as the case may be. Notice that the two terms have the same denominator $n$ because $\Pi_{n+1}^{n}+U_{n+1}^{n}$ is a value scaled down from $n+1$ to $n$. If a policyholder joins a stock insurance company, he or she owes only $\Pi_{n} / n$ or $\Pi_{n+1}^{n} / n$. $U_{n} / n$ and $U_{n+1}^{n} / n$ are one-time membership fee that a policyholder has to pay before joining a mutual insurance company. Hence the advantage of a stock insurer from the viewpoint of policyholders is that initial payment is cheaper without the membership fee.

Given this advantage, how can mutual insurance companies compete with stock insurers in the insurance market? Numerous organizational forms coexist in the market because each has its unique "survival value" (Fama and Jensen [8]). Weak evidence is witnessed that a mutual insurer is more efficient than a stock insurer (Mayers and Smith [11]). 
But more importantly major advantages of a mutual from the viewpoint of policyholders are: (i) policyholders can protect their wealth from other interest groups (Mayers and Smith [10]); (ii) policyholders receive the full benefit of diversification; and (iii) better quality insurance is easily obtainable after the growth of an underwriting portfolio. These advantages are further discussed in the following subsections.

\section{Policyholder wealth protection}

From theorem 3, after the expansion of an underwriting portfolio, shareholders of a stock insurer receive shareholder dividends, and policyholders pay higher $\Pi$. Consequently the wealth of policyholders will transfer to shareholders. In the case of a mutual insurer, however, policyholder contribution (premium and capital) remains the same before and after the growth. Thus the wealth of policyholders is fully protected.

\section{Benefit of diversification without extra cost}

For a stock insurance company, the benefit of diversification (i. e., improved solvency probability and dividend payment in the context of the article) goes to shareholders. Policyholders have to pay an extra charge for the improved solvency probability from diversification. In the case of a mutual insurer, owner policyholders fully enjoy this benefit without paying more. Besides, as the underwriting portfolio grows, per policyholder membership fee decreases because $U_{n+1}^{n} / n<U_{n} / n$, making it more affordable to purchase insurance from a mutual insurer. Given this fact, how can a small mutual insurer coexist with a large mutual insurer? One may find an analogy between this issue and the issue of scale economies in insurance. Detailed discussion of the subject, therefore, is well beyond the scope of the paper.

\section{Better quality insurance}

Expected profit of a stock insurer is given by

$$
\int_{0}^{\Pi+U}(\Pi-L) h(L) d L-U \int_{\Pi+U}^{\infty} h(L) d L,
$$

where $\Pi$ and $U$ are not necessarily identical to $\Pi$ and $U$ in (4). If the insurance company is solvent, profit or loss is the difference between $\Pi$ and $L$; when insolvent, owners lose $U$. Expected profit is simply the sum of the expectations of the two elements. In a perfectly competitive market, it becomes zero. Therefore,

$$
0=-U+\int_{0}^{\Pi+U} H(L) d L
$$

which is equivalent to equation (4). From theorems 1 and 2 , to provide better quality insurance, the relative contribution of shareholders compared to policyholders should increase with expected profit for shareholders unchanged at zero dollars. Naturally shareholders have no incentives to supply capital beyond the required minimum. In the absence of regulation, theorems 1 and 2 imply that shareholders will supply no capital, which is consistent with the results of Doherty [6]. 
Because the case of Figure 2(b) does not improve solvency probability after the expansion, discussion in this subsection is limited to the case of Figure 2(a). From Figure 2(a), expansion of the underwriting portfolio increases solvency probability from $A A^{\prime}$ to $B B^{\prime}$. A profit-maximizing stock insurer will hold only $\$ X$, although some conservative policyholders want better quality insurance. Policyholders of a mutual insurer, though, may achieve the desire by keeping $\$(Y-X)$ within the insurer.

\section{Concluding remarks}

The effect of expanding the underwriting portfolio of an insurance company is examined in this study from the viewpoints of owners and policyholders. It is shown that owners receive the benefit from the expansion of an underwriting portfolio; growth of a portfolio costs policyholders more. Because general aggregate loss function is selected for the analysis, the conclusions drawn in this article can be applied more broadly. Since only short term solvency probability is considered in this study, further research may be extended to long term solvency probability of an insurance company. 


\section{REFERENCES}

1. BEARD, R.E., PENTIKAINEN, T., and PESONEN, E.: Risk Theory: The Stochastic Basis of Insurance, Chapman and Hall, 1984.

2. BLAZENKO, G.: "The economics of reinsurance", The Journal of Risk and Insurance, 53 (June 1986), 258-293.

3. BORCH, K.: "The utility concept applied to the theory of insurance", ASTIN Bulletin (1961), 245-255.

4. BORCH, K.: "Equilibrium in a reinsurance market", Econometrica, 30 (July 1962), 424-444.

5. BORCH, K.: "Do we need utility theory in insurance?", paper presented at the 12th Seminar of the European Group of Risk and Insurance Economists, Bruxelles, Belgium, September 23-25, 1985.

6. DOHERTY, N. A.: "On the capital structure of insurance firms", paper presented at the International Conference on Insurance Solvency, Philadelphia, Pennsylvania, June 18-20, 1986.

7. DROPKIN, L. B.: "Size of loss distributions in workmen's compensation insurance", Proceedings of the Casualty Actuarial Society, 51 (1964), 198-223.

8. FAMA, E.F., and JENSEN, M.C.: "Separation of ownership and control", The Journal of Law and Economics, 26 (June 1983), 301-325.

9. HECKMANN, P.E., and MEYERS, G.G.: "The calculation of aggregate loss distributions from claim frequency and claim count distributions", Proceedings of the Casualty Actuarial Society, 70 (1983), 22-61.

10. MAYERS, D., and SMITH, Jr., C.W.: "Contractual provisions, organizational structure, and conflict control in insurance markets”, Journal of Business, 54 (July 1981), 407-434.

11. MAYERS, D., and SMITH, Jr., C. W.: "Ownership structure and control: The mutualization of stock life insurance companies", Journal of Financial Economics, 16 (May 1986), 73-98.

12. MOSSIN, J.: "Aspects of rational insurance purchasing”, The Journal of Political Economy, 76 (July/August 1968), 553-568.

13. NIELSON, N.: "Capacity of the property-casualty insurance industry", The Journal of Risk and Insurance, 51 (September 1984), 393-411.

14. PENTIKAINEN, T.: "On the solvency of insurers: A survey on the aspects involved", paper presented at the International Conference on Insurance Solvency, Philadelphia, Pennsylvania, June $18-20,1986$.

15. SAMSON, D., and THOMAS, H.: "Reinsurance decision making and expected utility", The Journal of Risk and Insurance, 50 (June 1983), 249-264.

16. VENEZIAN, E.C.: "Efficiency and equity in insurance", The Journal of Risk and Insurance, 51 (June 1984), 190-204.

17. WITT, R.C.: "Pricing and underwriting risk in automobile insurance: A probabilistic view", The Journal of Risk and Insurance, 40 (December 1973), 509-531.

18. WITT, R. C.: "Pricing, investment income, and underwriting risk: A stochastic view", The Journal of Risk and Insurance, 41 (March 1974), 109-133. 